Article

\title{
DRL1, Encoding A NAC Transcription Factor, Is Involved in Leaf Senescence in Grapevine
}

\author{
Ziguo Zhu ${ }^{1}$, Guirong $\mathrm{Li}^{2}$, Chaohui Yan ${ }^{2}$, Li Liu ${ }^{1}$, Qingtian Zhang ${ }^{1}$, Zhen Han ${ }^{1}$ and Bo Li ${ }^{1}$,* \\ 1 Shandong Institute of Pomology, Shandong Academy of Agricultural Science, No 66 Longtan Road, \\ Taian 271000, China; shanhong98@163.com (Z.Z.); 15153871569@163.com (L.L.); tcszqt@163.com (Q.Z.); \\ hanzhen86@163.com (Z.H.) \\ 2 College of Horticulture and Landscape Architecture, Henan Institute of Science and Technology, \\ Xinxiang 453003, China; liguirong10@163.com (G.L.); ychputao@163.com (C.Y.) \\ * Correspondence: sdtalibo@163.com; Tel.: +86-0538-8237362
}

Received: 7 May 2019; Accepted: 29 May 2019; Published: 31 May 2019

check for updates

\begin{abstract}
The NAC (for NAM, ATAF1,2, and CUC2) proteins family are plant-specific transcription factors, which play important roles in leaf development and response to environmental stresses. In this study, an NAC gene, DRL1, isolated from grapevine Vitis vinifera L. "Yatomi Rose", was shown to be involved in leaf senescence. The quantity of DRL1 transcripts decreased with advancing leaf senescence in grapevine. Overexpressing the DRL1 gene in tobacco plants significantly delayed leaf senescence with respect to chlorophyll concentration, potential quantum efficiency of photosystem II $(\mathrm{Fv} / \mathrm{Fm})$, and ion leakage. Moreover, exogenous abscisic acid (ABA) markedly reduced the expression of DRL1, and the ABA and salicylic acid (SA) concentration was lower in the DRL1-overexpressing transgenic plants than in the wild-type plants. The DRL1 transgenic plants exhibited reduced sensitivity to ABA-induced senescence but no significant change in the sensitivity to jasmonic acid-, SA- or ethylene-induced senescence. Transcriptomic analysis and RNA expression studies also indicated that the transcript abundance of genes associated with ABA biosynthesis and regulation, including 9-cis-epoxycarotenoid dioxygenase (NCED1), NCED5, zeaxanthin epoxidase1 (ZEP1), ABA DEFICIENT2 (ABA2), ABA4, and ABA INSENSITIVE 2 (ABI2), was markedly reduced in the $D R L 1$-overexpressing plants. These results suggested that $D R L 1$ plays a role as a negative regulator of leaf senescence by regulating ABA synthesis.
\end{abstract}

Keywords: Grapevine; NAC transcription factor; ABA; leaf senescence

\section{Introduction}

Aging is one of the most important physiological phenomena in biology, and in plants, the leaf is one of the organs most sensitive to senescence. Leaf senescence is not a passive degradation process, but a developmentally programmed process, regulated in an orderly fashion $[1,2]$. During leaf senescence, there are considerable changes in cell structure, hormone level, physiology, biochemical metabolism, and gene regulation [1].

Plant hormones play an important regulatory role in leaf senescence [1]. Cytokinins can inhibit leaf senescence and prolong leaf life while overexpression of isopentenyl-transferase (IPT), the key gene for cytokinin synthesis, can delay plant senescence [3,4]. Abscisic acid (ABA) can promote plant senescence with exogenous $\mathrm{ABA}$ application inducing the expression of senescence-associated genes (SAGs) and ABA-synthesis genes such as NCED2, NCED3, AAO1, and AAO3 in Arabidopsis thaliana (L.) Heynh and can lead to premature leaf senescence [5-7]. Ethylene and jasmonic acid (JA) can also promote plant senescence, and genes involved with the biosynthesis of ethylene (ET) and jasmonic acid (JA) were up-regulated following the exogenous application of the respective hormone $[8,9]$. 
Both the ethylene-insensitive mutants etr 1 and ein 2 and the JA-insensitive mutants coil of $A$. thaliana showed a delayed-aging phenotype [10-12]. Salicylic acid (SA) plays a role in promoting natural leaf senescence with $A$. thaliana plants defective in the SA-signaling pathway (namely npr1 and pad4 mutants) exhibiting reduced expression of SAGs and delayed-senescence phenotypes [13]. However, the effects of auxins or gibberellins (GA) on leaf senescence are not clear.

Leaf senescence is a complex developmental phase. The onset and progression of leaf senescence are controlled by an array of signal transduction genes [2]. NAC (NAM, ATAF1,2, and CUC2)-domain proteins are plant-specific transcription factors [14-16]. In the genomes of $A$. thaliana, Populus trichocarpa L., Glycine max L., and Oryza sativa L., 117, 120, 152, and 151 NAC genes have been identified [17-20], respectively. NAC proteins contain a highly conserved $\mathrm{N}$-terminal domain that is responsible for DNA binding activity and a variable C-terminal domain that ensures the specificity of NAC transactivation activity [15]. Many members of the NAC transcription factor family have been reported to be functionally involved in the regulation of leaf senescence in A. thaliana [21,22], wheat [23], bamboo [24], and rice [25]. For example, the $A$. thaliana mutant (anac092/atanc2/ore1) exhibited delayed leaf senescence [21]. AtNAP is strongly up-regulated during leaf senescence in $A$. thaliana, and atnap null mutants show a delayed leaf senescence phenotype, whereas the inducible overexpression of AtNAP causes precocious leaf senescence [22]. In wheat, overexpressing TaNAC-S resulted in delayed leaf senescence associated with increased grain yield and protein concentrations [23], whereas in bamboo, constitutive overexpression of $B e N A C 1$ resulted in precocious senescence phenotypes in A. thaliana [24], and overexpression of OsNAC2 dramatically accelerated leaf senescence in Oryza sativa [25].

Grapevine (Vitis vinifera L.) is an important fruit crop cultivated worldwide [26]. A total of 74 NAC genes were identified from the grapevine genome [27]. Several members of NAC in grapevine have been found to participate in development, abiotic, and biotic stress. Over-expression of VvNAC1 in A. thaliana enhanced tolerance to osmotic, salt, and cold stresses and to Botrytis cinerea and Hyaloperonospora arabidopsidis pathogens [28]. NAC26 from $V$. Amurensis showed the tolerance to drought stress by the regulation of JA in $A$. thaliana [29]. A different $V v N A C 26$ polymorphism and their combination were involved in berry size variation in grapevine [30]. However, the detailed function of grapevine NAC genes in terms of their role in leaf senescence is unknown. In our previous study, a NAC transcription factor gene DRL1 (GenBank No. XP-002281816) was identified from grapevine which was associated with dwarf plant and rolled leaf phenotypes [31]. In the present study, we report that DRL1 was involved in leaf senescence, and the regulatory mechanism might be associated to the ABA signaling pathway.

\section{Results}

\subsection{DRL1 is Down-Regulated during Leaf Senescence in Grapevine}

Leaf senescence seriously affects photosynthesis and nutrient assimilation, and subsequently influences the yield and quality of grapes [32,33]. To confirm the role of the DRL1 gene in leaf development, we determined its transcript abundance at different stages of leaf development in grapevine. The expression level of the DRL1 gene was highest in the young leaf, decreasing with leaf age, and reaching the lowest level in leaves at late senescence (Figure 1a,b). In other tissues, including root, stem flower, and fruit, the transcripts of DRL1 kept to a low level (Figure 1b). Transgenic tobacco plants were generated, which expressed the GUS protein driven by the promoter of the DRL1 gene. A histochemical staining analysis showed that the DRL1 gene was mainly expressed in the stem and leaves (Figure 1c). In 14-day-old transgenic plants, more GUS protein was detected in the young leaf, which showed dark blue, whereas less GUS protein was detected in the old leaf, which showed only slightly blue. A similar result was obtained in 4-week-old transgenic plants (Figure 1d). These results show that the DRL1 gene may be involved in leaf senescence. 


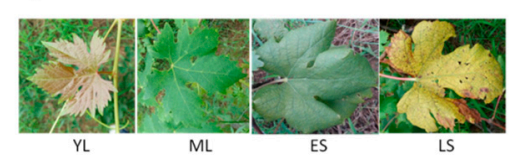

b

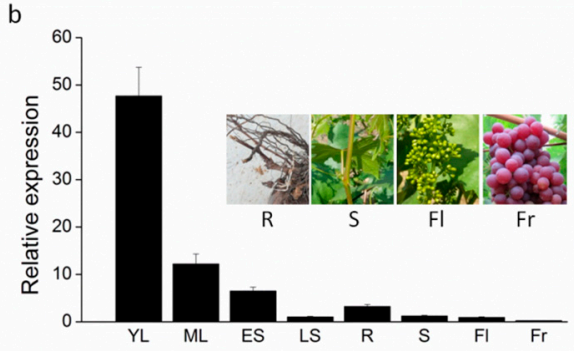

C

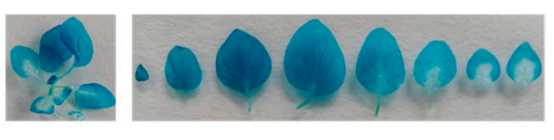

d

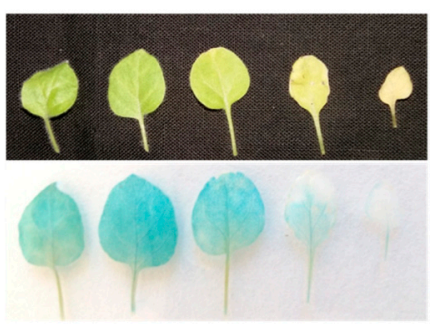

Figure 1. The expression of DRL1 during leaf senescence in grapevine. (a) Leaf senescence phenotypes in grapevine cultivar 'Yatomi Rosa'. YL, expanding young leaf; ML, mature leaf that is fully expanded but with no visible yellowing; ES, early senescence leaf, with about $5 \%$ of the leaf area yellowing; LS, late senescence leaf, with more than $50 \%$ of the leaf area yellowing. (b) Relative expression of DRL1 during leaf senescence. The expression levels were determined using qPCR. The expression levels in LS leaves were set to 1 . Bars represent mean \pm standard deviation (SD). (c, d) GUS staining in leaves of

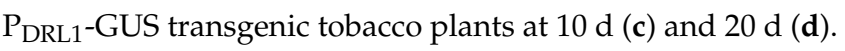

\subsection{Ectopic Overexpression of DRL1 Delayed Leaf Senescence}

We confirmed the role of $D R L 1$ in leaf senescence by monitoring leaf color as a marker of senescence. Wild-type (WT) tobacco plants exhibited the visible yellowing phenotype in older leaves compared with the leaf at the same stage of development in similar-aged plants of the DRL1-overexpressing transgenic plants (Figure 2a). In 10-week-old plants, the survival rate of leaves in the transgenic plants was higher than that in WT plants, the survival rate being $55.6 \%$ in transgenic plants, while only $39.1 \%$ in WT tobacco plants (Figure 2b, Figure S1c,e). In 12-week-old plants, there was still a 30.2\% survival rate of leaves in the transgenic plants, but no leaves (green or yellow) remained on the WT plants (Figure 2b). Further physiological analyses of leaf senescence were performed on 8-week-old tobacco plants. The delay in leaf senescence in the transgenic plants compared to their WT counterparts was also supported by changes in total chlorophyll (Chl) concentration (Figure 2c), ion leakage (Figure 2e), and the potential quantum efficiency of photosystem II in the dark-adapted state ( $F v / F m)$ (Figure 2d). Moreover, DRL1 transgenic plants exhibited a delay in the plant height and the leaves curled inward compared with the wild type. To eliminate potential indirect effects of growth retardation on leaf senescence, the $12^{\text {th }}-14^{\text {th }}$ leaves of plants that exhibited similar patterns of leaf aging were assayed. After treatment in dark for $5 \mathrm{~d}$, WT plant leaves retained $65 \%$ of their chlorophyll concentration, while transgenic plants retained $82 \%$ of their $\mathrm{Chl}$ concentration. Hence, the retardation and curled leaves did not affect the function of leaf senescence (Figure S1,2). These results suggest that DRL1 negatively regulates leaf senescence. 
a

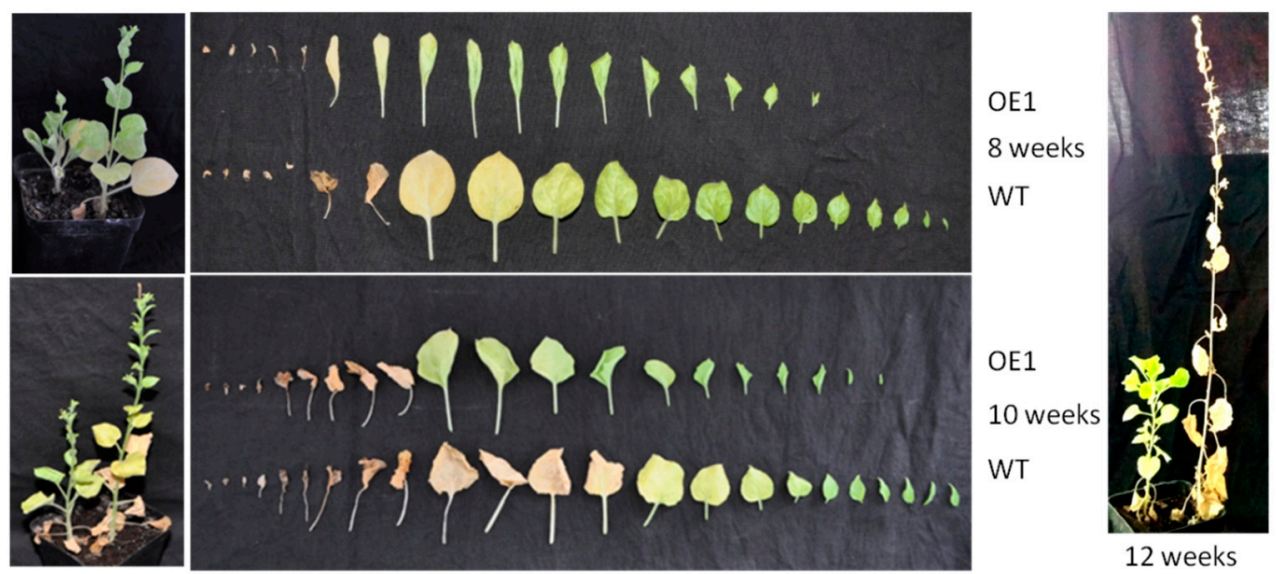

b

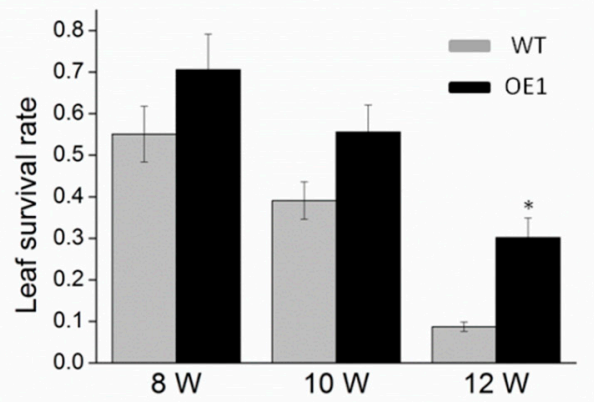

d

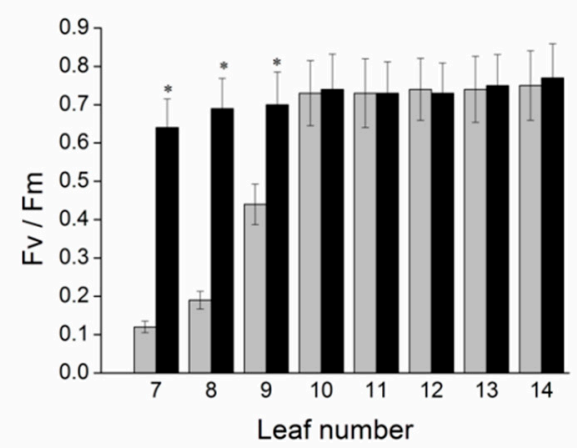

C

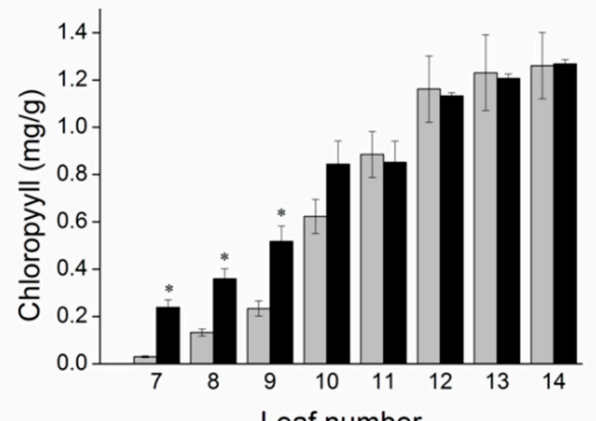

e

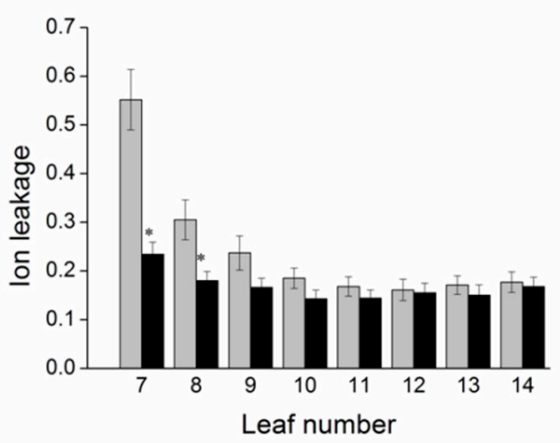

Figure 2. DRL1-over-expression delayed leaf senescence. (a) The phenotype of transgenic and wild-type (WT) tobacco plants at 8, 10, and 12 weeks. (b-e) Physiological analyses of leaves from transgenic plants. Leaf survival rate (b), chlorophyll concentration (c), ion leakage (d), and $F v / F m$ ratio (e) were measured in WT and transgenic plants of the same age. Bars represent mean \pm SD. Significant difference from the WT was confirmed by Tukey's test $[34,35]\left({ }^{*} p<0.05\right)$.

\subsection{Analysis of Differentially Expressed Genes (DEGs) in DRL1-Overexpressing Transgenic Plants}

To investigate the possible regulatory roles of $D R L 1$ at the transcriptional level, we generated cDNA libraries from leaves of DRL1 transgenic and WT tobacco plants and performed the genome-wide expression profile analysis using the Illumina platform (Table S1). In the whole unigene set, a total of 44265 unigenes were significantly matched to known genes in the public databases of NR, NT, Swiss-Prot, KEGG, COG, and GO (Table S2). A total of 1149 differentially expressed genes (DEGs) were screened out using the combined criteria of at least a two-fold change and a significant chi-square test $(p<0.05$, false detection rate $(F D R)<0.01)$. Among these DEGs, 584 were up-regulated and 565 were down-regulated in the transgenic plant relative to the WT (Figure 3a, Figure S3). According to 
GO annotation and category, these DEGs were involved in regulating gene, development, transport, stress, carbohydrate metabolism, degradation, and secondary metabolism, and unknown gene for the biological process categories, of which the largest number of sequences were those associated with regulatory gene pathways (except for unknown genes) (Figure $3 b$ ).

a

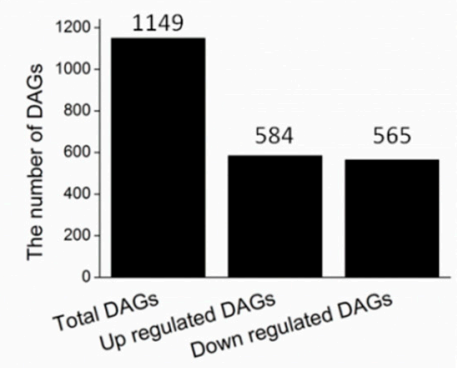

C

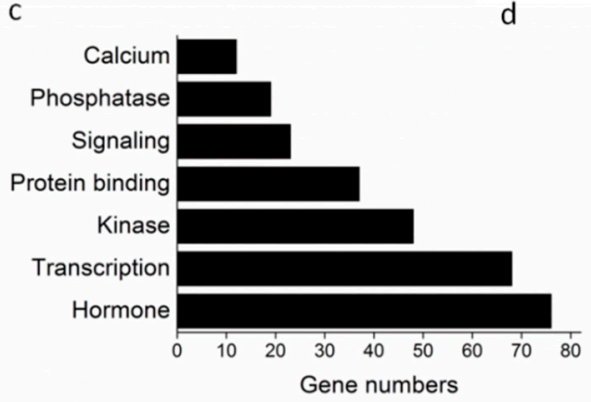

b
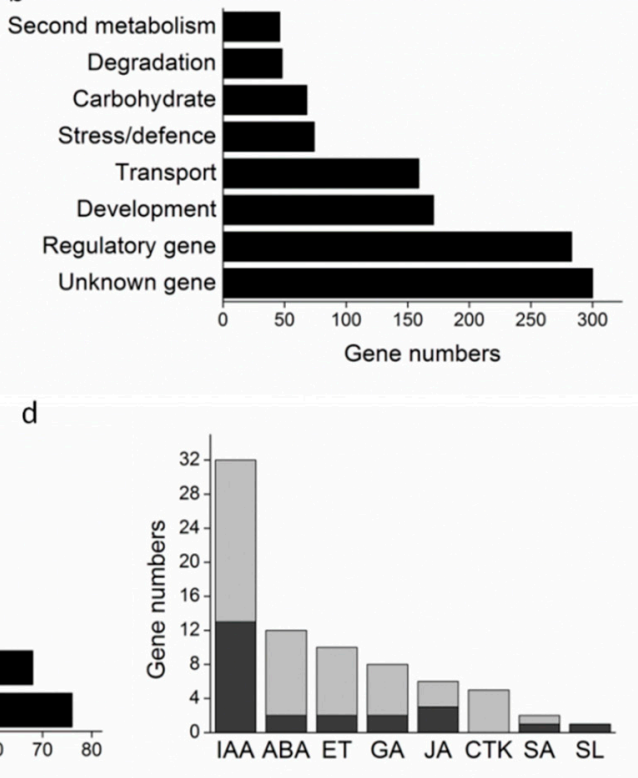

Figure 3. DRL1 regulates various genes associated with leaf senescence. (a) The number of DEGs in DRL1-overexpressing transgenic plants compared with wild type plants. (b) Classification of DAGs based on Gene Ontology (GO) annotation. (c) Classification of regulatory genes. (d) Classification of hormone-related genes (gray columns represent up-regulated genes, black columns represent down-regulated genes).

To further characterize the regulatory genes, DEGs of the regulatory gene category were further subdivided into seven subgroups, including hormone, transcription, kinase, protein-binding, signaling, phosphatase, and calcium. The largest number of regulatory sequences was associated with hormones (Figure 3c, Table S3). Among these hormone-related genes, the largest number of genes were those related to auxin (IAA) and ABA. The IAA-related genes included 13 up-regulated genes and 19 down-regulated genes, while the ABA-related genes contained two up-regulated genes and ten down-regulated genes (Figure 3d). We speculated that IAA and ABA play a key role in leaf senescence in the DRL1-overexpressing transgenic tobacco plants.

\subsection{ABA Is Involved in Leaf Senescence of DRL1-Overexpressing Transgenic Plants}

To further analyze the role of the hormone ABA in leaf senescence, we investigated the hormone concentration in the transgenic and WT tobacco plants. The results are shown in Figure 4. Among the six major plant hormones, there was no significant change in auxins (IAA, IBA, ICA, ME-IAA), cytokinins (cZ, TZ, DZ, IP), gibberellins, or jasmonic acid between DRL1-overexpressing transgenic and WT plants. However, the concentrations of salicylic acid (SA) and ABA in the transgenic plants were significantly lower, with reductions of $32.8 \%$ and $75.4 \%$, respectively, compared with WT plants. 

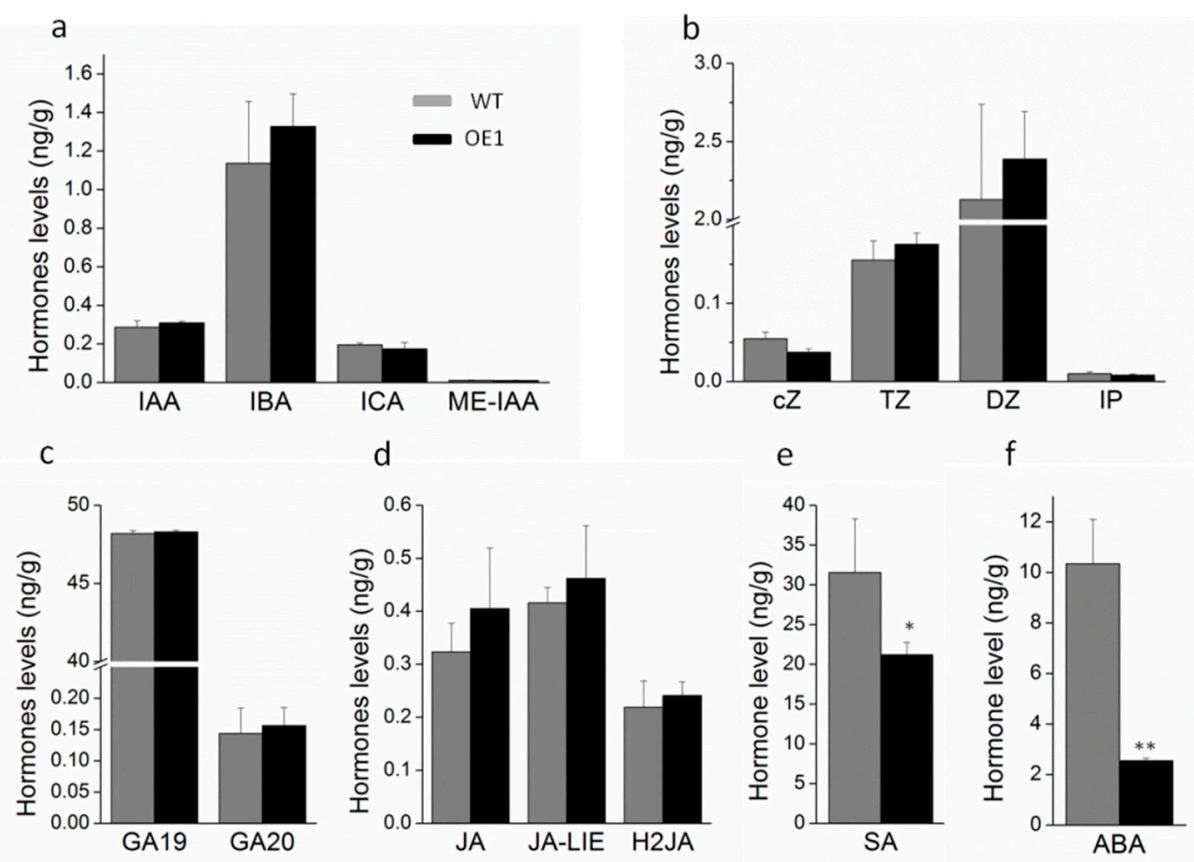

Figure 4. The concentrations of the hormones auxins (a), cytokinins (b), gibberellins (c), jasmonic acid (d), salicylic acid (e), and abscisic acid (f) in DRL-overexpressing transgenic and wild-type tobacco leaves. Bars represent mean \pm SD. Significant difference from the WT was confirmed by Tukey's test (*p $p 0.05$ and $\left.{ }^{* *} p<0.01\right)$.

To test whether DRL1 is involved in ABA-induced leaf senescence, we compared the senescence response of 8-week-old leaves of both transgenic and WT plants following exogenous ABA treatment. By $9 \mathrm{~d}$ after ABA treatment, WT leaves had lost most of their Chl concentration (Figure 5a). In contrast, the leaves of the transgenic plants had retained $30 \%$ of their Chl concentration (Figure $5 b$ ). We then carried out the same experiment using detached leaf disks (Figure 5c). After $7 \mathrm{~d}$ of ABA treatment, WT leaf disks retained $50 \%$ of the Chl concentration retained by the disk of the transgenic plants. In addition, we tested whether DRL1 was also involved in the leaf senescence response to other hormones (Figure 5d). After $9 \mathrm{~d}$ of exogenous MeJA, SA, or ETH treatments, there was no significant difference in leaf color between transgenic and WT plants, both of which had lost most of their Chl concentrations and only retained $11 \%$ of their Chl concentration. Moreover, IAA, GA, and CTK did not affect leaf senescence by the regulation of DRL1 (Figure S4). 
a

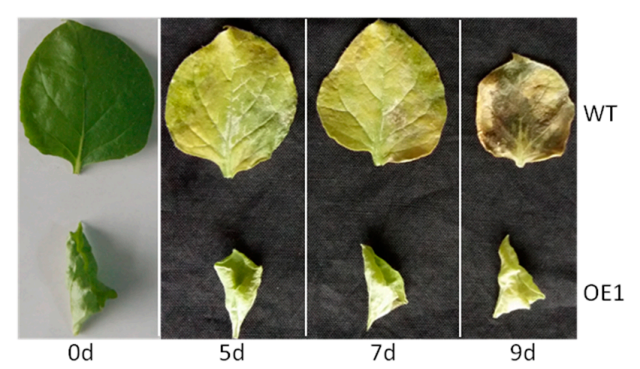

C

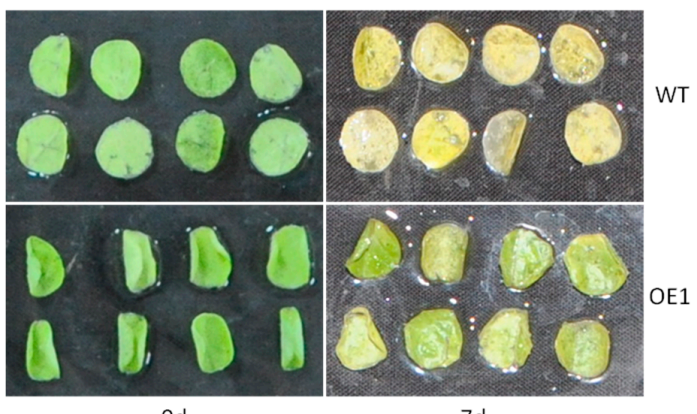

od

e

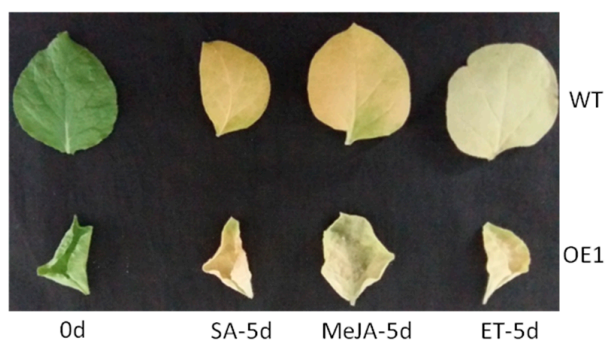

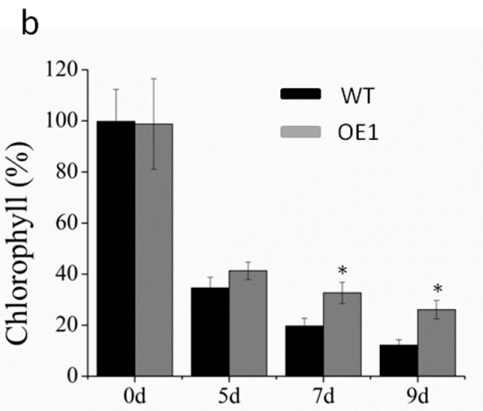

d

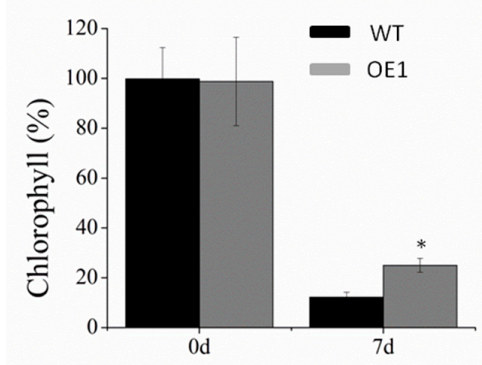

f

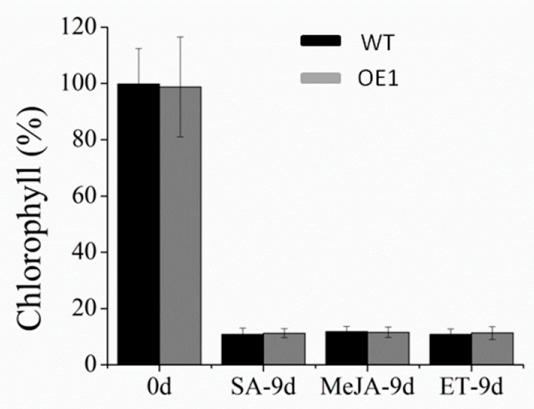

Figure 5. Leaf senescence induction by application of hormones to DRL1-overexpressing transgenic tobacco plants. $(\mathbf{a}, \mathbf{b})$ Detached leaf senescence phenotypes of transgenic tobacco treated with abscisic acid (a) and determination of chlorophyll concentration (b). (c, d) Leaf senescence phenotypes of transgenic tobacco leaf disks treated with ABA (c) and determination of chlorophyll content (d). (e) Leaf senescence phenotypes of transgenic tobacco treated with salicylic acid, methyl jasmonate, or ethylene followed by determination of chlorophyll content (f). Bars represent mean \pm SD with at least three biological replicates. Significant difference from the WT was confirmed by Tukey's test $\left({ }^{*} p<0.05\right)$.

\subsection{DRL1 Delayed Leaf Senescence by Regulation of ABA}

To better understand the regulatory mechanism of DRL1 in the process of leaf senescence, we investigated the expression profile of DRL1 in response to exogenous application of the hormone ABA in grapevine. The expression of DRL1 decreased following treatment with ABA. The transcripts showed a strong reduction of $99.9 \%$ at $48 \mathrm{~h}$ after treatment with ABA compared with the control (Figure 6a). The promoter-inducible activity was assessed using GUS histochemical staining in transgenic plants. Following treatment with water (Control check, CK), 7-day-old transgenic seedlings were heavily stained blue, whereas in plants treated with ABA, only the leaf margins were stained blue, and they were only lightly stained (Figure 6b). To understand how DRL1 controls leaf senescence at the molecular level, we examined the genes related to ABA biosynthesis and regulation (Figure $6 \mathrm{c}$ ). The ABA biosynthesis genes were NCED and ZEP1. The expressions of NCED1, NCED2, NCED3, NCED5, and ZEP1 were significantly down-regulated in transgenic tobacco leaves compared with the WT. In particular, NCED3 and NCED5 transcripts in transgenic tobacco showed remarkable reductions, 
$95.1 \%$ and $93.8 \%$, respectively, compared with WT. With respect to the ABA regulatory genes, the transcripts of $A B A 2, A B A 4, A B I 2$, and PYL4 were also suppressed in the transgenic plants, with $75.7 \%$, $60.3 \%, 43.7 \%$, and $89.9 \%$ reductions, respectively, compared with the WT. These results indicated that ABAs play an important role in leaf senescence.

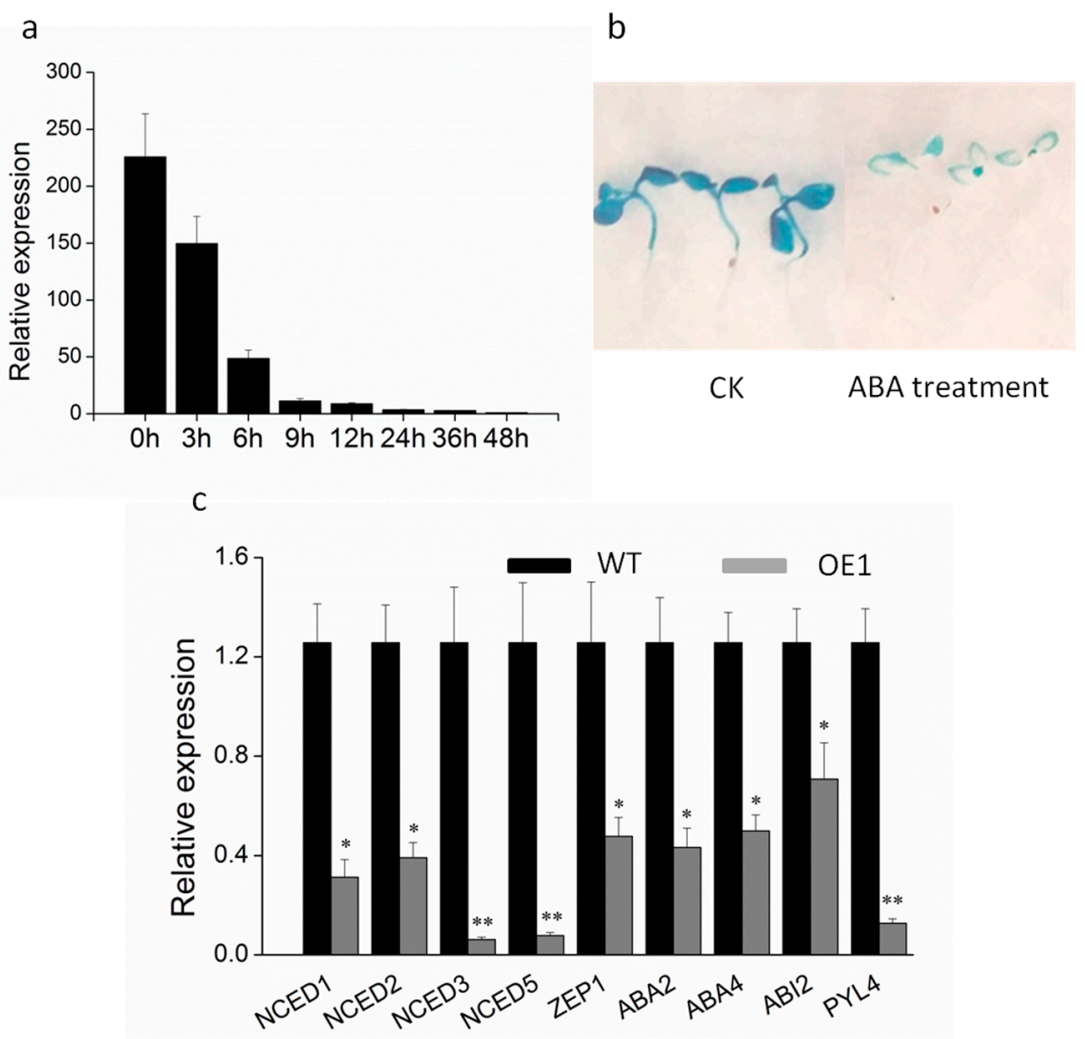

Figure 6. The expression of abscisic acid metabolism-related genes in WT and DRL1-overexpressing transgenic plants. (a) The expression of DRL1 in grapevine following ABA treatment. (b) GUS staining in leaves of $P_{D R L 1}$-GUS transgenic tobacco plants under ABA treatment. Six-day-old tobacco seedlings were treated $(n=60)$. (c) Expression analysis of ABA biosynthesis- and regulation-related genes in wild-type and DRL1-overexpressing transgenic tobacco plants. Asterisks represent statistically significant differences between wild-type and transgenic plants: ${ }^{*} p<0.05$ and ${ }^{* *} p<0.01$.

\section{Discussion}

Leaf senescence is a complex developmental process, the regulation of which involves many highly organized molecular and cellular processes $[1,36]$. The $A$. thaliana transcriptome showed that about $10 \%$ of the genes were differentially expressed during the late developmental period of leaf senescence, including 134 genes encoding transcription factors such as NAC, WRKY, AP2/EREBP, and MYB [37-39]. Of the transcription factors, the NAC family is particularly rich in senescence-regulated transcription factors in many plant species [40,41]. In A. thaliana, approximately 20 NAC genes have been shown to exhibit increased expression in senescing leaves [42], while in Gossypium hirsutum L., the NAC proteins GhNAC11, GhNAC20, GhNAC57, and GhNAC78 undergo expression changes during leaf senescence [43]. In our study, RT-qPCR showed the opposite expression pattern of DRL1, which was down-regulated during natural leaf senescence. The result of histochemical staining was also consistent with the RT-qPCR data, with DRL1 being mainly expressed in adult leaves and a lower level in old and yellowing leaves, indicating that DRL1 might be involved in a negative manner in leaf senescence.

Most NAC genes related to leaf senescence play a positive role, such as AtNAP [22], NAM-B1 [44], NTL4 [45], and ANAC092/AtNAC2/ORE1 [46]. A few studies have concerned a negative role for 
NAC genes in leaf senescence. The A. thaliana NAC transcription factor JUNGBRUNNEN1 and $V N I 2$ negatively regulate leaf senescence $[47,48]$. In wheat, overexpression of TaNAC-S resulted in delayed leaf senescence [23]. To better understand the role of DRL1 in leaf senescence, physiological analyses of the DRL1-overexpressing transgenic plants were performed. Tobacco is easily transformed using Agrobacterium and usually as a model plant organism for studying fundamental biological processes [49,50]; hence, we choose tobacco for the functional analysis of DRL1. Compared with the WT tobacco plants, leaves exhibiting over-expression of DRL1 displayed significantly increased Chl concentration, markedly increased Fv/Fm ratios, and reduced levels of ion leakage. Chl concentration is an indicator of plant nutrition, photosynthesis ability, and developmental stage, while the Fv/Fm ratio is an indicator of the photochemical quantum efficiency of PSII [51]. We predicted that DRL1 might act on leaf senescence as a negative regulator.

Leaf senescence is regulated by both internal and external factors. Internal factors include leaf age, developmental stage, and endogenous hormone levels, which, in turn, are controlled by regulatory genes [52]. Transcriptome analysis of DRL1-overexpressing transgenic plants showed that the largest number of DEGs (except for the unknown gene) corresponded to the regulatory genes $(24.6 \%)$, of which $26.8 \%$ were related to hormones. Plant hormones play a key role in plant growth and development, as well as the response to external stimuli. Comparing the hormone concentrations in transgenic and WT tobacco plants, there was no significant change in auxin, cytokinin, gibberellin, or jasmonic acid concentrations, but concentrations of both SA and ABA were significantly reduced in the transgenic plants. Previous studies reported that SA, ET, and ABA can promote leaf senescence. In A. thaliana, overexpression of the transcription factor gene WRKY 75 accelerated leaf senescence by up-regulating the genes related to the SA signal pathway [53]. The A. thaliana mitochondrial protease FtSH4 induced leaf senescence via up-regulation of the WRKY-dependent salicylic acid signal pathway [54]. To determine whether reduced SA and ABA concentrations were the only reason behind the delayed leaf senescence of DRL1-overexpressing transgenic plants, we investigated the senescence process in transgenic leaves treated with different hormones. The results supported the hypothesis that ABA plays a key role in DRL1-regulated leaf senescence, as the ABA-treated plants retained more $\mathrm{Chl}$ than those treated with exogenous SA, ET, and MeJA. Like $A$. thaliana NAC transcription factor VNI2 [48], grapevine DRL1 delayed leaf senescence in tobacco by mediating ABA signals.

It is well known that ABA participates in leaf senescence. Some SAGs are induced by ABA and then promote plant senescence, including SAG113 [55], AtNAP [22], and VNI2 [48]. DRL1 showed an expression pattern opposite to that of SAGs, with a marked decrease in response to ABA application, and the results were also obtained from DRL1 promoter analysis. The expression of RAV1 was also repressed by ABA, but constitutive expression in A. thaliana caused premature leaf senescence [56]. Endogenous ABA concentration increases significantly in many plants during leaf senescence. In Solanum lycopersicum L., the ABA concentration increased linearly with time during leaf senescence [57]. In Zea mays L., the ABA concentration in "stay-green" phenotype varieties was significantly higher than that in premature-senescence varieties, which may be the reason for the delayed senescence in "stay-green" varieties [51]. These results indicated that regulation of ABA biosynthesis plays a key role in leaf senescence.

In the present study, expression analyses also confirmed that overexpression of DRL1 caused drastic down-regulation of ABA biosynthetic genes, with abundance of NCED1, NCED2, NCED3, NCED5 and ZEP1 transcripts all being notably suppressed. NCEDs are the key enzymes controlling ABA production, being involved in xanthophyll cleavage [58]. ZEP catalyzes the conversion of zeaxanthin into violaxanthin in ABA biosynthesis [59]. Ectopic expression of the rice NAC transcription factor gene $O s N A C 2$ led to an increase in ABA levels via directly upregulating the expression of the ABA biosynthetic genes NCED3 and ZEP1 [60]. SINAP2 promotes leaf senescence in tomato by directly controlling the expression of genes for ABA biosynthesis, such as genes encoding NCED1, ABC transporter $\mathrm{G}$ family member 40 , and $\mathrm{ABA} 8^{\prime}$-hydroxylase [61]. Moreover, the transcript levels of the regulatory genes, $A B A 2, A B A 4$, and $A B I$ were also sharply reduced in DRL1-overexpressing 
transgenic tobacco plants. ABA2 encodes a short-chain dehydrogenase/reductase 1 that catalyzes the multi-step conversion of xanthoxinto abscisic aldehyde during ABA biosynthesis in A. thaliana [62]. Transgenic plants overexpressing $A t A B A 2$ have been shown to increase ABA concentration, promote seed dormancy, and increase stress tolerance [63]. The $A$. thaliana mutant aba4 showed reduced endogenous ABA concentration in rosettes and seeds [64], while the A. thaliana mutant abi2 showed higher ABA concentration in plants and sensitivity to ABA [65]. Therefore, we speculated that DRL1 might delay leaf senescence by down-regulation of ABA biosynthesis.

\section{Materials and Methods}

\subsection{Plant Material and Hormone Treatment}

V. vinifera cv. "Yatomi Rose" was grown in the grape germplasm resource orchard of Shandong Institute of Pomology, Taian, Shandong, China. Leaves at different stages of development were collected from the upper, middle, and lower part of the branch. One-year-old rooted cuttings of $V$. vinifera $\mathrm{cv}$. Yatomi Rose were grown in a greenhouse for hormone treatments. The grapevine cuttings were sprayed with $100 \mu \mathrm{M}$ abscisic acid (ABA) containing $0.05 \%$ Tween 20 [66]. Leaves sprayed with $0.05 \%$ Tween 20 solution were used as a negative control. Leaves were harvested at $0,3,6,9,12,24$, 36 , and $48 \mathrm{~h}$ after treatment with ABA, and immediately frozen in liquid nitrogen. Leaves from at least three replicate plants were collected and pooled to constitute one replicate for each time point, with three biological replicates for each treatment. To examine hormone-induced leaf senescence, the 6-week-old tobacco leaves were floated on $3 \mathrm{mM}$ 2-Morpholinoethanesulfonic Acid (MES) buffer (pH 5.8) containing $50 \mathrm{mM} \mathrm{ABA}, 50 \mu \mathrm{M} \mathrm{SA}, 50 \mathrm{mM}$ MeJA, or $5 \mathrm{mM}$ ethylene. All chemical treatments were performed at $22{ }^{\circ} \mathrm{C}$ under continuous lighting. All stress-induction experiments were performed on three independent replicate plants for each treatment.

\subsection{RNA Analysis}

Total RNA was extracted using the method described by Zhang et al. [67], and first-strand cDNA was synthesized from $2 \mu \mathrm{g}$ of total RNA at $42{ }^{\circ} \mathrm{C}$ with PrimeScript RT Reagent Kit (Takara, Dalian, China). All PCRs were performed on a Bio-Rad IQ-5 thermocycler (Bio-Rad Laboratories, Berkeley, CA, USA) with 40 cycles of $5 \mathrm{~s}$ at $94{ }^{\circ} \mathrm{C}$ and $30 \mathrm{~s}$ at $58^{\circ} \mathrm{C}$. Cycle threshold values were determined by IQ-5 Bio-Rad software assuming 100\% primer efficiency. All primers are listed in Table 1. Three mRNA samples from three independently harvested replicate leaf samples were qPCR analyzed. The relative mRNA ratios were calculated as $2^{-\Delta \Delta C T}$ [68]. The transcript levels of target genes were normalized against VvActin for the grape samples and NtActin for Nicotiana samples [69,70].

Table 1. Primers in the experiment for PCR.

\begin{tabular}{|c|c|c|}
\hline Gene Name & Forward Primer $5^{\prime} \rightarrow 3^{\prime}$ & Reverse Primer $5^{\prime} \rightarrow 3^{\prime}$ \\
\hline NtNCED1 (NM_001325669) & gtttggtggggagcctctgttttta & tgatttccattctttctcattgtgc \\
\hline NtNCED2 (NM_001326185) & tccacttcaaaaccaaccactatt & ttaaggcactttccacggcatcta \\
\hline NtNCED3 (JX101473) & ctcacacgaactccaaaacccactt & tagcaccattacggacataaacccc \\
\hline NtNCED5 (XM_016625112) & ctccaaaacccacttcctaaaaca & agcaccattacggacataaacccc \\
\hline NtZEP1 (XM_016582459) & tagaggaccaattcagatacagagca & agttaccagaaacaccatcaaccaag \\
\hline NtABA2 (EU123520) & ggtgctgatggcataaggtctaa & ctccacccacatctgaagaaaca \\
\hline NtABA4 (XM_016637325) & cttccacttgcttttgtcactccc & cacccatttctatgcttacttccc \\
\hline NtABI2 (NM_001324946) & tgttatgcagggtggtgttaaaggc & aaggtgaaggtgaaagagatgatgt \\
\hline NtPYL4 (XP_00977852) & atcggtctgttactactttacatcct & cctttcgtctacccaaattctca \\
\hline NtActin (XM_016618073) & tggagaaaatctggcatcacacg & actggcataaagggatagaacgg \\
\hline VvDRL1 (XP-002281816) & ccgggtttcggtttcatccta & actccetctcgccaatcttcg \\
\hline VvActin (AF369524) & cctcaaccccaaggccaacaga & accatcaccagaatccagcaca \\
\hline
\end{tabular}




\subsection{Production of Transgenic Tobacco}

DNA from the grapevine leaves was extracted using the CTAB (Cetyltrimethylammonium bromide) method. The DRL1 promoter was amplified using the PrimerSTAR GXL DNA Polymerase (Takara, Dalian, China) with the forward primer $5^{\prime}$-GATGTTTGAAATATTTTTAAA TAAGTA-3' and reverse primer $5^{\prime}$-GTCTACTGCTTACTGGTGTTGTCCTGTGA-3'. The PCR reaction had the following thermal profile: $94^{\circ} \mathrm{C}$ for $3 \mathrm{~min}, 28$ cycles of $94^{\circ} \mathrm{C}$ for $30 \mathrm{~s}, 60^{\circ} \mathrm{C}$ for $30 \mathrm{~s}$, and $72{ }^{\circ} \mathrm{C}$ for $60 \mathrm{~s}$, followed by $72{ }^{\circ} \mathrm{C}$ for $10 \mathrm{~min}$. The 1140 -bp promoter of DRL1 was inserted into the vector pCAMIA1391z to generate the construct $D R L 1_{\text {pro }}$ :GUS. The transformation of tobacco was performed as described by Horsch et al. [49]. The selection of the transformed plants was conducted by culturing the plantlets on MS medium containing hygromycin $\left(25 \mathrm{mg} \mathrm{L}^{-1}\right)$ under $14 \mathrm{~h}$ of light and $10 \mathrm{~h}$ of dark. The $\mathrm{T}_{1}$ generation of transgenic tobacco was used for further analysis.

\subsection{Chlorophyll Concentration, Fluorescence, and Ion Leakage Analysis}

The tobacco plants were placed in a plant culture room at $25-28^{\circ} \mathrm{C}, 14 \mathrm{~h}$ light $/ 10 \mathrm{~h}$ dark, $2000 \mathrm{Lx}$ (Illumination intensity). Chlorophyll of $0.5 \mathrm{~g}$ leaves was extracted using $80 \%$ acetone and analyzed using a UV-Visible Spectrophotometer (UV2600, Shimadzu, Kyoto, Japan) at 645 and $653 \mathrm{~nm}$ [70]. Relative electrolyte leakage was assessed by ion leakage analysis as previously described by Zhang [57]. Potential quantum efficiency for PSII (Fv/Fm) was measured as described by Jiang [71].

\subsection{Transcriptome Analysis}

Transgenic tobacco plants and the WT plants were divided into two groups, each with two independent biological replicates. Mature leaves collected from the middle of the tobacco plants were used for total RNA isolation. The differentially expressed gene (DEG) experiments were performed by the Biomarker Technology Company (Beijing, China). Total reads were generated using the HisSeq $^{\mathrm{TM}} 2500$. The raw data were first purified by trimming adapters and removing low quality sequencing. All adaptor-trimmed reads were mapped to the tobacco genome (ftp://ftp.solgenomics. net/genomes/Nicotiana-tabacum/) using a Basic Local Alignment Search Tool (BLAST)-like alignment tool (BLAT). For the differentially expressed genes assay, FPKM (fragments per kilobase per million reads) values were used to normalize gene expression levels [72]. DEGS between the transgenic and WT plants were screened using DESeq software [73], based on a false discovery rate (FDR) $<0.01$ and $\mid \log _{2}$ (fold change) $\mid>1$ or $<-1$. For the annotation and categorization, the DEGs were subjected to the GO database (http://www.geneontology.org/) and the Kyoto Encyclo- pedia of Genes and Genomes (KEGG) database (http://www.genome.jp/kegg/pathway.html) using Blast2GO and KEGG Automatic Annotation Server (KAAS, http://www.genome.jp/kegg/kaas/), respectively. For the categorization, the DEGs were analyzed for GO category enrichment and KEGG pathway enrichment using GO-seq and KAAS, respectively [74].

\subsection{Hormones Analysis}

For transgenic and WT tobacco plants, leaves in the middle of the plant from 6-week-old OE1 (DRL1-overexpressing plants) and WT were harvested under normal conditions. The hormones were extracted and quantified by MetWare (http://www.metware.cn/) based on a LC-MS/MS platform (MetWare Company, Wuhan, China). Three biological replicates of each assay were performed.

\section{Conclusions}

Our study demonstrated that DRL1 gene from grapevine plays a negative role in leaf senescence. Overexpression of DRL1 gene in tobacco plants significantly delayed leaf senescence. Moreover, transcriptomics and hormone analysis indicated that the transcript abundance of genes were associated with ABA biosynthesis. The exogenous hormones treatment to transgenic plants and RNA expression analysis further confirmed that DRL1 delayed leaf senescence by regulation of ABA signal pathway. 
But which gene is the direct target gene of the DRL1, NECD, ZEP, ABA, ABI, or other genes? This will be our future research which will be helpful to know the regulation mechanism of leaf senescence in grapevine.

Supplementary Materials: Supplementary materials can be found at http://www.mdpi.com/1422-0067/20/11/ 2678/s1.

Author Contributions: Z.Z. and B.L. conceived and designed the experiments. Z.Z. and C.Y. conducted most of the experiments. G.L. participated in the experiments. L.L., Q.Z. and Z.H. contributed materials and analysis tools. Z.Z. wrote the paper. All authors read and approved the final manuscript.

Funding: This work was supported by Shandong Major Science and Technology Project (Grant No. 2016ZDJS10A01), Shandong Forestry Science and Technology Innovation Project (Grant No. LYCX03-2018-13) and China's Post-doctoral Science Foundation (Grant No. 2016M592231).

Conflicts of Interest: The authors declare that they have no conflict of interests.

\section{Abbreviations}

$\begin{array}{ll}\text { ABA } & \text { Abscisic acid } \\ \text { Chl } & \text { Chlorophyll } \\ \text { CTAB } & \text { Cetyltrimethylammonium bromide } \\ \text { DEGs } & \text { Differentially expressed genes } \\ \text { GUS } & \beta \text {-glucuronidase } \\ \text { JA } & \text { Jasmonic acid } \\ \text { NCED } & \text { 9-cisepoxycarotenoid dioxygenase } \\ \text { SA } & \text { Salicylic acid } \\ \text { ZEP } & \text { Zeaxanthin epoxidase }\end{array}$

\section{References}

1. Lim, P.O.; Kim, H.J.; Nam, H.G. Leaf senescence. Annu. Rev. Plant Biol. 2007, 58, 115-136. [CrossRef]

2. Guo, Y.; Gan, S. Leaf senescence: Signals, execution, and regulation. Curr. Top Dev. Biol. 2005, 71, 83-112. [PubMed]

3. Kant, S.; Burch, D.; Badenhorst, P.; Palanisamy, R.; Mason, J.; Spangenberg, G. Regulated expression of a cytokinin biosynthesis gene IPT delays leaf senescence and improves yield under rainfed and irrigated conditions in canola (Brassica napus L.). PLoS ONE 2015, 10, e0116349. [CrossRef]

4. Robson, P.R.; Donnison, I.S.; Wang, K.; Frame, B.; Pegg, S.E.; Thomas, A.; Thomas, H. Leaf senescence is delayed in maize expressing the Agrobacterium IPT gene under the control of a novel maize senescence-enhanced promoter. Plant Biotechnol. J. 2004, 2, 101-112. [CrossRef]

5. Van der Graaff, E.; Schwacke, R.; Schneider, A.; Desimone, M.; Flugge, U.I.; Kunze, R. Transcription analysis of Arabidopsis membrane transporters and hormone pathways during developmental and induced leaf senescence. Plant Physiol. 2006, 141, 776-792. [CrossRef]

6. Jibran, R.; Hunter, D.A.; Dijkwel, P.P. Hormonal regulation of leaf senescence through integration of developmental and stress signals. Plant Mol. Biol. 2013, 82, 547-561. [CrossRef] [PubMed]

7. Osborne, D.J. Hormonal regulation of leaf senescence. Symp. Soc. Exp. Biol. 1967, 21, 305-321. [PubMed]

8. He, Y.; Fukushige, H.; Hildebrand, D.F.; Gan, S. Evidence supporting a role of jasmonic acid in Arabidopsis leaf senescence. Plant Physiol. 2002, 128, 876-884. [CrossRef] [PubMed]

9. Li, Z.; Guo, H. Ethylene Treatment in studying leaf senescence in Arabidopsis. Methods Mol. Biol. 2018, 1744, 105-112.

10. Qiu, K.; Li, Z.; Yang, Z.; Chen, J.; Wu, S.; Zhu, X.; Gao, S.; Gao, J.; Ren, G.; Kuai, B.; et al. EIN3 and ORE1 accelerate degreening during ethylene-mediated leaf senescence by directly activating chlorophyll catabolic genes in Arabidopsis. PLoS Genet 2015, 11, e1005399. [CrossRef]

11. Li, Z.; Peng, J.; Wen, X.; Guo, H. Ethylene-insensitive3 is a senescence-associated gene that accelerates age-dependent leaf senescence by directly repressing miR164 transcription in Arabidopsis. Plant Cell 2013, 25, 3311-3328. [CrossRef]

12. Lee, S.H.; Sakuraba, Y.; Lee, T.; Kim, K.W.; An, G.; Lee, H.Y.; Paek, N.C. Mutation of Oryza sativa CORONATINE INSENSITIVE $1 \mathrm{~b}$ (OsCOI1b) delays leaf senescence. J. Integr. Plant Biol. 2015, 57, 562-576. [CrossRef] 
13. Morris, K.; MacKerness, S.A.; Page, T.; John, C.F.; Murphy, A.M.; Carr, J.P.; Buchanan-Wollaston, V. Salicylic acid has a role in regulating gene expression during leaf senescence. Plant J. 2000, 23, 677-685. [CrossRef]

14. Quattrocchio, F.; Wing, J.; van der Woude, K.; Souer, E.; de Vetten, N.; Mol, J.; Koes, R. Molecular analysis of the anthocyanin2 gene of petunia and its role in the evolution of flower color. Plant Cell 1999, 11, 1433-1444. [CrossRef]

15. Riechmann, J.L.; Heard, J.; Martin, G.; Reuber, L.; Jiang, C.; Keddie, J.; Adam, L.; Pineda, O.; Ratcliffe, O.J.; Samaha, R.R.; et al. Arabidopsis transcription factors: Genome-wide comparative analysis among eukaryotes. Science 2000, 290, 2105-2110. [CrossRef]

16. Souer, E.; vanHouwelingen, A.; Kloos, D.; Mol, J.; Koes, R. The no apical meristem gene of petunia is required for pattern formation in embryos and flowers and is expressed at meristem and primordia boundaries. Cell 1996, 85, 159-170. [CrossRef]

17. Hu, R.; Qi, G.; Kong, Y.; Kong, D.; Gao, Q.; Zhou, G. Comprehensive analysis of NAC domain transcription factor gene family in Populus trichocarpa. BMC Plant Biol. 2010, 10, 145. [CrossRef]

18. Li, C.W.; Su, R.C.; Cheng, C.P.; Sanjaya; You, S.J.; Hsieh, T.H.; Chao, T.C.; Chan, M.T. Tomato RAV transcription factor is a pivotal modulator involved in the AP2/EREBP-mediated defense pathway. Plant Physiol. 2011, 156, 213-227. [CrossRef]

19. Nuruzzaman, M.; Manimekalai, R.; Sharoni, A.M.; Satoh, K.; Kondoh, H.; Ooka, H.; Kikuchi, S. Genome-wide analysis of NAC transcription factor family in rice. Gene 2010, 465, 30-44. [CrossRef]

20. Ooka, H.; Satoh, K.; Doi, K.; Nagata, T.; Otomo, Y.; Murakami, K.; Matsubara, K.; Osato, N.; Kawai, J.; Carninci, P.; et al. Comprehensive analysis of NAC family genes in Oryza sativa and Arabidopsis thaliana. DNA Res. 2003, 10, 239-247. [CrossRef]

21. Oh, S.K.; Lee, S.; Yu, S.; Choi, D. Expression of a novel NAC domain-containing transcription factor (CaNAC1) is preferentially associated with incompatible interactions between chili pepper and pathogens. Planta 2005, 222, 876-887. [CrossRef]

22. Guo, Y.; Gan, S. AtNAP, a NAC family transcription factor, has an important role in leaf senescence. Plant J. 2006, 46, 601-612. [CrossRef]

23. Zhao, D.; Derkx, A.P.; Liu, D.C.; Buchner, P.; Hawkesford, M.J. Overexpression of a NAC transcription factor delays leaf senescence and increases grain nitrogen concentration in wheat. Plant Biol. 2015, 17, 904-913. [CrossRef]

24. Chen, Y.; Qiu, K.; Kuai, B.; Ding, Y. Identification of an NAP-like transcription factor BeNAC1 regulating leaf senescence in bamboo (Bambusa emeiensis "Viridiflavus"). Physiol. Plant 2011, 142, 361-371. [CrossRef] [PubMed]

25. Mao, C.; Lu, S.; Lv, B.; Zhang, B.; Shen, J.; He, J.; Luo, L.; Xi, D.; Chen, X.; Ming, F. A rice NAC transcription factor promotes leaf senescence via ABA biosynthesis. Plant Physiol. 2017, 174, 1747-1763. [CrossRef]

26. Jaillon, O.; Aury, J.M.; Noel, B.; Policriti, A.; Clepet, C.; Casagrande, A.; Choisne, N.; Aubourg, S.; Vitulo, N.; Jubin, C.; et al. The grapevine genome sequence suggests ancestral hexaploidization in major angiosperm phyla. Nature 2007, 449, 463-467. [PubMed]

27. Wang, N.; Zheng, Y.; Xin, H.; Fang, L.; Li, S. Comprehensive analysis of NAC domain transcription factor gene family in Vitis vinifera. Plant Cell Rep. 2013, 32, 61-75. [CrossRef] [PubMed]

28. Le Henanff, G.; Profizi, C.; Courteaux, B.; Rabenoelina, F.; Gerard, C.; Clement, C.; Baillieul, F.; Cordelier, S.; Dhondt-Cordelier, S. Grapevine NAC1 transcription factor as a convergent node in developmental processes, abiotic stresses, and necrotrophic/biotrophic pathogen tolerance. J. Exp. Bot. 2013, 64, 4877-4893. [CrossRef]

29. Fang, L.; Su, L.; Sun, X.; Li, X.; Sun, M.; Karungo, S.K.; Fang, S.; Chu, J.; Li, S.; Xin, H. Expression of Vitis amurensis NAC26 in Arabidopsis enhances drought tolerance by modulating jasmonic acid synthesis. J. Exp. Bot. 2016, 67, 2829-2845. [CrossRef] [PubMed]

30. Tello, J.; Torres-Perez, R.; Grimplet, J.; Carbonell-Bejerano, P.; Martinez-Zapater, J.M.; Ibanez, J. Polymorphisms and minihaplotypes in the VvNAC26 gene associate with berry size variation in grapevine. BMC Plant Biol. 2015, 15, 253. [CrossRef]

31. Yan, C.; Li, G.; Mu, J.; Lou, H.; Zhu, Z. Subcellular localization and functional analysis of a NAC gene VvDRL1 from Vitis vinifera "Yatomi Rosa". Acta Horticulturae Sinica 2016, 43, 643-652.

32. Diago, M.P.; Correa, C.; Millan, B.; Barreiro, P.; Valero, C.; Tardaguila, J. Grapevine yield and leaf area estimation using supervised classification methodology on RGB images taken under field conditions. Sensors 2012, 12, 16988-17006. [CrossRef] 
33. Wu, X.Y.; Kuai, B.K.; Jia, J.Z.; Jing, H.C. Regulation of leaf senescence and crop genetic improvement. J. Integ. Plant Biol. 2012, 54, 936-952. [CrossRef]

34. Antonello, J.M.; Clark, R.L.; Heyse, J.F. Application of the tukey trend test procedure to assess developmental and reproductive toxicity. I. Measurement data. Fundam. Appl. Toxicol. 1993, 21, 52-58. [CrossRef]

35. Moulia-Pelat, J.P.; Spiegel, A.; Baudon, D.; Merlin, M.; Lemardeley, P.; Daumerie, D.; Gateff, C. Epidemiological value of graphic representation of the Tukey test. Med. Trop. (Mars) 1989, 49, 285-288.

36. Zhou, Y.; Huang, W.; Liu, L.; Chen, T.; Zhou, F.; Lin, Y. Identification and functional characterization of a rice NAC gene involved in the regulation of leaf senescence. BMC Plant Biol. 2013, 13, 132. [CrossRef]

37. Hou, K.; Wu, W.; Gan, S.S. SAUR36, a small auxin up RNA gene, is involved in the promotion of leaf senescence in Arabidopsis. Plant Physiol. 2013, 161, 1002-1009. [CrossRef]

38. Gan, S.; Amasino, R.M. Inhibition of leaf senescence by autoregulated production of cytokinin. Science 1995, 270, 1986-1988. [CrossRef]

39. He, Y.; Gan, S. A gene encoding an acyl hydrolase is involved in leaf senescence in Arabidopsis. Plant Cell 2002, 14, 805-815. [CrossRef]

40. Gregersen, P.L.; Holm, P.B. Transcriptome analysis of senescence in the flag leaf of wheat (Triticum aestivum L.). Plant Biotechnol. J. 2007, 5, 192-206. [CrossRef]

41. Balazadeh, S.; Riano-Pachon, D.M.; Mueller-Roeber, B. Transcription factors regulating leaf senescence in Arabidopsis thaliana. Plant Biol. (Stuttg) 2008, 10 Suppl. 1, 63-75. [CrossRef]

42. Guo, Y.; Cai, Z.; Gan, S. Transcriptome of Arabidopsis leaf senescence. Plant Cell Environ. 2004, 27, 521-549. [CrossRef]

43. Shah, S.T.; Pang, C.; Fan, S.; Song, M.; Arain, S.; Yu, S. Isolation and expression profiling of GhNAC transcription factor genes in cotton (Gossypium hirsutum L.) during leaf senescence and in response to stresses. Gene 2013, 531, 220-234. [CrossRef] [PubMed]

44. Uauy, C.; Distelfeld, A.; Fahima, T.; Blechl, A.; Dubcovsky, J. A NAC Gene regulating senescence improves grain protein, zinc, and iron content in wheat. Science 2006, 314, 1298-1301. [CrossRef] [PubMed]

45. Lee, S.; Seo, P.J.; Lee, H.J.; Park, C.M. A NAC transcription factor NTL4 promotes reactive oxygen species production during drought-induced leaf senescence in Arabidopsis. Plant J. 2012, 70, 831-844. [CrossRef] [PubMed]

46. Balazadeh, S.; Siddiqui, H.; Allu, A.D.; Matallana-Ramirez, L.P.; Caldana, C.; Mehrnia, M.; Zanor, M.I.; Kohler, B.; Mueller-Roeber, B. A gene regulatory network controlled by the NAC transcription factor ANAC092/AtNAC2/ORE1 during salt-promoted senescence. Plant J. 2010, 62, 250-264. [CrossRef] [PubMed]

47. Wu, A.; Allu, A.D.; Garapati, P.; Siddiqui, H.; Dortay, H.; Zanor, M.I.; Asensi-Fabado, M.A.; Munne-Bosch, S.; Antonio, C.; Tohge, T.; et al. JUNGBRUNNEN1, a reactive oxygen species-responsive NAC transcription factor, regulates longevity in Arabidopsis. Plant Cell 2012, 24, 482-506. [CrossRef] [PubMed]

48. Yang, S.D.; Seo, P.J.; Yoon, H.K.; Park, C.M. The Arabidopsis NAC transcription factor VNI2 integrates abscisic acid signals into leaf senescence via the COR/RD genes. Plant Cell 2011, 23, 2155-2168. [CrossRef]

49. Horsch, R.B.; Fry, J.E.; Hoffmann, D.; Eichholtz, D.; Rogers, S.G.; Fraley, R.T. A simple and general method for transferring genes into plants. Science 1985, 227, 1229-1231.

50. Sierro, N.; Battey, J.N.; Ouadi, S.; Bakaher, N.; Bovet, L.; Willig, A.; Goepfert, S.; Peitsch, M.C.; Ivanov, N.V. The tobacco genome sequence and its comparison with those of tomato and potato. Nat. Commun. 2014, 5, 3833. [CrossRef] [PubMed]

51. He, P.; Osaki, M.; Takebe, M.; Shinano, T.; Wasaki, J. Endogenous hormones and expression of senescence-related genes in different senescent types of maize. J. Exp. Bot. 2005, 56, 1117-1128. [CrossRef] [PubMed]

52. Lim, P.O.; Nam, H.G. The molecular and genetic control of leaf senescence and longevity in Arabidopsis. Curr. Top Dev. Biol. 2005, 67, 49-83. [PubMed]

53. Guo, P.; Li, Z.; Huang, P.; Li, B.; Fang, S.; Chu, J.; Guo, H. A Tripartite amplification loop involving the transcription factor WRKY75, salicylic acid, and reactive oxygen species accelerates leaf senescence. Plant Cell 2017, 29, 2854-2870. [CrossRef] [PubMed]

54. Zhang, S.; Li, C.; Wang, R.; Chen, Y.; Shu, S.; Huang, R.; Zhang, D.; Li, J.; Xiao, S.; Yao, N.; et al. The Arabidopsis mitochondrial protease FtSH4 is involved in leaf senescence via regulation of WRKY-Dependent salicylic acid accumulation and signaling. Plant Physiol. 2017, 173, 2294-2307. [CrossRef] 
55. Zhang, K.; Xia, X.; Zhang, Y.; Gan, S.S. An ABA-regulated and Golgi-localized protein phosphatase controls water loss during leaf senescence in Arabidopsis. Plant J. 2012, 69, 667-678. [CrossRef]

56. Woo, H.R.; Kim, J.H.; Kim, J.; Lee, U.; Song, I.J.; Lee, H.Y.; Nam, H.G.; Lim, P.O. The RAV1 transcription factor positively regulates leaf senescence in Arabidopsis. J. Exp. Bot. 2010, 61, 3947-3957. [CrossRef]

57. Ghanem, M.E.; Albacete, A.; Martinez-Andujar, C.; Acosta, M.; Romero-Aranda, R.; Dodd, I.C.; Lutts, S.; Perez-Alfocea, F. Hormonal changes during salinity-induced leaf senescence in tomato (Solanum lycopersicum L.). J. Exp. Bot. 2008, 59, 3039-3050. [CrossRef] [PubMed]

58. Tan, B.C.; Joseph, L.M.; Deng, W.T.; Liu, L.; Li, Q.B.; Cline, K.; McCarty, D.R. Molecular characterization of the Arabidopsis 9-cis epoxycarotenoid dioxygenase gene family. Plant J. 2003, 35, 44-56. [CrossRef]

59. Nambara, E.; Marion-Poll, A. Abscisic acid biosynthesis and catabolism. Annu. Rev. Plant Biol. 2005, 56, 165-185. [CrossRef]

60. Ma, X.; Zhang, Y.; Tureckova, V.; Xue, G.P.; Fernie, A.R.; Mueller-Roeber, B.; Balazadeh, S. The NAC transcription factor SINAP2 regulates leaf senescence and fruit yield in tomato. Plant Physiol. 2018, 177, 1286-1302. [CrossRef]

61. Gonzalez-Guzman, M.; Apostolova, N.; Belles, J.M.; Barrero, J.M.; Piqueras, P.; Ponce, M.R.; Micol, J.L.; Serrano, R.; Rodriguez, P.L. The short-chain alcohol dehydrogenase ABA2 catalyzes the conversion of xanthoxin to abscisic aldehyde. Plant Cell 2002, 14, 1833-1846. [CrossRef] [PubMed]

62. Lin, P.C.; Hwang, S.G.; Endo, A.; Okamoto, M.; Koshiba, T.; Cheng, W.H. Ectopic expression of ABSCISIC ACID 2/GLUCOSE INSENSITIVE 1 in Arabidopsis promotes seed dormancy and stress tolerance. Plant Physiol. 2007, 143, 745-758. [CrossRef]

63. North, H.M.; De Almeida, A.; Boutin, J.P.; Frey, A.; To, A.; Botran, L.; Sotta, B.; Marion-Poll, A. The Arabidopsis ABA-deficient mutant aba4 demonstrates that the major route for stress-induced ABA accumulation is via neoxanthin isomers. Plant J. 2007, 50, 810-824. [CrossRef]

64. Leung, J.; Merlot, S.; Giraudat, J. The Arabidopsis ABSCISIC ACID-INSENSITIVE2 (ABI2) and ABI1 genes encode homologous protein phosphatases 2C involved in abscisic acid signal transduction. Plant Cell 1997, 9, 759-771. [CrossRef]

65. Xiao, H.; Nassuth, A. Stress- and development-induced expression of spliced and unspliced transcripts from two highly similar dehydrin 1 genes in V. riparia and V. vinifera. Plant Cell Rep. 2006, 25, 968-977. [CrossRef] [PubMed]

66. Zhang, Z.; Huang, R. Enhanced tolerance to freezing in tobacco and tomato overexpressing transcription factor TERF2/LeERF2 is modulated by ethylene biosynthesis. Plant Mol. Biol. 2010, 73, 241-249. [CrossRef] [PubMed]

67. Livak, K.J.; Schmittgen, T.D. Analysis of relative gene expression data using real-time quantitative PCR and the 2(-Delta Delta C(T)) Method. Methods 2001, 25, 402-408. [CrossRef] [PubMed]

68. Reid, K.E.; Olsson, N.; Schlosser, J.; Peng, F.; Lund, S.T. An optimized grapevine RNA isolation procedure and statistical determination of reference genes for real-time RT-PCR during berry development. BMC Plant Biol. 2006, 6, 27. [CrossRef]

69. Gutha, L.R.; Casassa, L.F.; Harbertson, J.F.; Naidu, R.A. Modulation of flavonoid biosynthetic pathway genes and anthocyanins due to virus infection in grapevine (Vitis vinifera L.) leaves. BMC Plant Biol. 2010, 10, 187. [CrossRef]

70. Costa, M.D.; Reis, P.A.; Valente, M.A.; Irsigler, A.S.; Carvalho, C.M.; Loureiro, M.E.; Aragao, F.J.; Boston, R.S.; Fietto, L.G.; Fontes, E.P. A new branch of endoplasmic reticulum stress signaling and the osmotic signal converge on plant-specific asparagine-rich proteins to promote cell death. J. Biol. Chem. 2008, 283, 20209-20219. [CrossRef]

71. Jiang, G.; Wang, Z.; Shang, H.; Yang, W.; Hu, Z.; Phillips, J.; Deng, X. Proteome analysis of leaves from the resurrection plant Boea hygrometrica in response to dehydration and rehydration. Planta 2007, 225, 1405-1420. [CrossRef]

72. Trapnell, C.; Williams, B.A.; Pertea, G.; Mortazavi, A.; Kwan, G.; van Baren, M.J.; Salzberg, S.L.; Wold, B.J.; Pachter, L. Transcript assembly and quantification by RNA-Seq reveals unannotated transcripts and isoform switching during cell differentiation. Nat. Biotechnol. 2010, 28, 511-515. [CrossRef] [PubMed] 
73. Anders, S.; Huber, W. Differential expression analysis for sequence count data. Gen. Biol. 2010, 11, R106. [CrossRef]

74. Pervaiz, T.; Haifeng, J.; Salman Haider, M.; Cheng, Z.; Cui, M.; Wang, M.; Cui, L.; Wang, X.; Fang, J. Transcriptomic analysis of grapevine (cv. Summer Black) leaf, using the illumina platform. PloS ONE 2016, 11, e0147369. [CrossRef] 